\title{
Relic density in nonuniversal gaugino mass models with SO(10) GUT symmetry
}

\author{
Katri Huitu* \\ Department of Physics, and Helsinki Institute of Physics, \\ P.O. Box 64, FIN-00014 University of Helsinki, Finland \\ Jari Laamanen ${ }^{\dagger}$ \\ Institut für Physik, Technische Universität Dortmund, D-44221 Dortmund, Germany
}

(Dated: November 8, 2018)

\begin{abstract}
Nonuniversal boundary conditions in grand unified theories can lead to nonuniversal gaugino masses at the unification scale. In $R$-parity preserving theories the lightest supersymmetric particle is a natural candidate for the dark matter. The composition of the lightest neutralino and the identity of the next-to-lightest supersymmetric particle are studied, when nonuniversal gaugino masses come from representations of $\mathrm{SO}(10)$. In these cases, the thermal relic density compatible with the Wilkinson Microwave Anisotropy Probe observations is found. Relic densities are compared with the universal case. Mass spectra in the studied cases are discussed.
\end{abstract}

PACS numbers: 12.60.Jv, 95.35.+d

Keywords: Gaugino masses, relic density, dark matter

\section{INTRODUCTION}

The phenomenology of supersymmetric models depends crucially on the compositions of neutralinos and charginos, if the lightest neutralino is the lightest supersymmetric particle (LSP). In addition to the laboratory studies, relevant input is obtained from the dark matter searches, where the the Wilkinson Microwave Anisotropy Probe (WMAP) satellite has put precise limits on the relic density. Supersymmetric theories which preserve $R$-parity contain a natural candidate for the cold dark matter particle. Neutralino LSP can provide the appropriate relic density.

In many supergravity type models the lightest neutralino is binolike, which often leads to too large thermal relic density, as compared to the limits provided by the WMAP experiment [1]. When the gaugino masses are not universal at the grand unification scale, the resulting neutralino composition changes from the case of universal gaugino masses [2]. In this paper, the thermal relic density of the neutralino LSP is studied, when gaugino masses are due to nonuniversal representations of $\mathrm{SO}(10)$ grand unified theory (GUT) $[3,4]$. Dark matter in a particular gauge symmetry breaking chain of the $\mathrm{SO}(10)$ GUT in the case of universal gaugino masses has been recently studied in [5]. Some phenomenological aspects of $\mathrm{SO}(10)$ GUTs with nonuniversal gaugino masses have been considered in $[6,7]$.

$\mathrm{SO}(10)$ has many attractive features among the GUT models. One of the most appealing properties is that one family of matter fermions can be put into a single 16-dimensional irreducible spinor representation of $\mathrm{SO}(10)$, including the right-handed neutrino $[8,9]$. In

\footnotetext{
*Electronic address: katri.huitu@helsinki.fi

${ }^{\dagger}$ Electronic address: jari.laamanen@uni-dortmund.de
}

addition, $\mathrm{SO}(10)$ allows possibility for the Yukawa coupling unification and representations are anomaly free. Conservation of $R$-parity, which forbids the unwanted dimension-five operators leading to rapid proton decay, may result from the $\mathrm{SO}(10)$ symmetry breaking. The doublet-triplet splitting could be achieved using, e.g. , the so-called Dimopoulos-Wilczek mechanism [10]. Because the $\mathrm{SO}(10)$ gauge symmetry breaks down to the standard model (SM) gauge symmetry through some intermediate group, the $\mathrm{SO}(10)$ GUT offers several possibilities for the model building. For example, it can contain as a subgroup the Pati-Salam $\mathrm{SU}(4) \times \mathrm{SU}(2) \times \mathrm{SU}(2)$ model.

Gaugino masses originate from the non-renormalizable terms in the $N=1$ supergravity Lagrangian involving the gauge kinetic function $f_{a b}(\Phi)$ [11]. The gauge part of the Lagrangian contains the gauge kinetic function coupling with two field strength superfields $W^{a}$. The Lagrangian for the coupling can be written as

$$
\mathcal{L}_{g k}=\int d^{2} \theta f_{a b}(\Phi) W^{a} W^{b}+\text { H.C. },
$$

where $a$ and $b$ are gauge group indices (for example, $a, b=1,2, \ldots, 45$ for $\mathrm{SO}(10))$, and repeated indices are summed over. The function $f_{a b}(\Phi)$ is an analytic function of the chiral superfields $\Phi$ in the theory. The chiral superfields $\Phi$ consist of a set of gauge singlet superfields $\Phi^{s}$ and gauge nonsinglet superfields $\Phi^{n}$ under the grand unified group. The gauge kinetic function $f_{a b}(\Phi)$ can be expanded,

$$
f_{a b}(\Phi)=f_{0}\left(\Phi^{s}\right) \delta_{a b}+\sum_{n} f_{n}\left(\Phi^{s}\right) \frac{\Phi_{a b}^{n}}{M_{P}}+\cdots
$$

where $\Phi^{s}$ and $\Phi^{n}$ are the singlet and nonsinglet chiral superfields, respectively. Here $f_{0}\left(\Phi^{s}\right)$ and $f_{n}\left(\Phi^{s}\right)$ are functions of gauge singlet superfields $\Phi^{s}$, and $M_{P}$ is some large scale. In order to generate a mass term for the gauginos, the gauge kinetic function must be non-minimal, 
i.e., it must not be a constant [12]. When $F_{\Phi}$ gets a vacuum expectation value (vev) $\left\langle F_{\Phi}\right\rangle$, the interaction (1) gives rise to gaugino masses:

$$
\mathcal{L}_{g k} \supset \frac{\left\langle F_{\Phi}\right\rangle_{a b}}{M_{P}} \lambda^{a} \lambda^{b}+\text { H.C. },
$$

where $\lambda^{a, b}$ are gaugino fields. The nonuniversal gaugino masses are generated by the nonsinglet chiral superfield $\Phi^{n}$ that appears linearly in the gauge kinetic function $f_{a b}(\Phi)$ in Eq. (2).

Gauginos belong to the adjoint representation of the gauge group, which in the case of $\mathrm{SO}(10)$ is the $\mathbf{4 5}$ dimensional representation. Because Eq. (3) must be gauge invariant, $\Phi$ and $F_{\Phi}$ must belong to some of the following representations appearing in the symmetric product of the two 45 dimensional representations of $\mathrm{SO}(10)$ $[13,14]$ :

$$
(45 \otimes \mathbf{4 5})_{S y m m}=\mathbf{1} \oplus \mathbf{5 4} \oplus \mathbf{2 1 0} \oplus \mathbf{7 7 0} .
$$

The representations 54,210 and $\mathbf{7 7 0}$ may lead to nonuniversal gaugino masses, while the $\mathbf{1}$ dimensional representation gives manifestly the universal gaugino masses. The relations between the gaugino masses are determined by the representation invariants, and are specific for each of the representations. Because the gauge kinetic function in Eq. (2) can get contributions from several different $\Phi$ 's, a linear combination of any of the representations is also possible. In that case the gaugino mass terms are not uniquely determined anymore, in contrast to the contribution purely from one representation. Here we assume that the dominant component of the gaugino masses comes from only one representation. This gives us a clear understanding of the role of different representations.

\section{DARK MATTER IN SO(10) REPRESENTATIONS}

\section{A. Breaking Chains: $\mathrm{SO}(10) \rightarrow H \rightarrow \mathrm{SM}$}

The GUT group SO(10) breaks down to the standard model gauge group $\mathrm{SU}(3) \times \mathrm{SU}(2) \times \mathrm{U}(1)$ via some intermediate gauge group $H$. Therefore the gaugino mass relations depend also on the gauge group breaking chain, in addition to the representation invariants coming from the gauge kinetic function. Moreover, the intermediate breaking scale affects also the generated gaugino masses via heavy gauge supermultiplets that correspond to the broken generators. However, if the gauge breaking from the GUT group to the SM group takes place at the GUT scale, these loop-induced messenger contributions [15] can be neglected in comparison to the tree-level contributions. Some fits to the experimental data in $\mathrm{SO}(10)$ GUT indicate that the two breaking scales are very close to each other, see [16, 17], although realistic models exist also with large splitting of the scales [18]. In this work we assume that the breaking from $\mathrm{SO}(10)$ to the SM gauge group happens at the GUT scale, and that the GUT breaking does not affect the gauge coupling unification.

We will study the representations 54 and 210 in the right-hand side of Eq. (4). The interesting breaking chains of 54 and 210 are included also in the breaking chains of $\mathbf{7 7 0}$. Table I shows possible SO(10) breaking chains $[14,19,20]$, which include the standard model gauge group, for the two chosen representations. Some of the subgroups lead to universal gaugino masses, or to massless gauginos [14], and we do not consider them. We will limit ourselves to the intermediate gauge groups $\mathrm{SU}(4) \times \mathrm{SU}(2) \times \mathrm{SU}(2), \mathrm{SU}(2) \times \mathrm{SO}(7)$ and $\mathrm{SU}(5) \times \mathrm{U}(1)$.

TABLE I: Breaking chains of $\mathrm{SO}(10)$ representations 54 and 210 which include the SM gauge group.

\begin{tabular}{ccc}
\hline \hline$F_{\Phi}$ & $H$ & Subgroup description \\
\hline \multirow{2}{*}{54} & $\mathrm{SU}(4) \times \mathrm{SU}(2) \times \mathrm{SU}(2)$ & Pati-Salam \\
& $\mathrm{SU}(2) \times \mathrm{SO}(7)$ & Universal gauginos \\
\hline & $\mathrm{SO}(9)$ & Massless gluino \\
\multirow{2}{*}{$\mathbf{2 1 0}$} & $\mathrm{SU}(4) \times \mathrm{SU}(2) \times \mathrm{SU}(2)$ & Massless $\mathrm{SU}(2) \mathrm{L}$ gauginos \\
& $\mathrm{SU}(3) \times \mathrm{SU}(2) \times \mathrm{SU}(2) \times \mathrm{U}(1)$ & \\
& $\mathrm{SU}(3) \times \mathrm{SU}(2) \times \mathrm{U}(1) \times \mathrm{U}(1)$ & "Flipped" $\mathrm{SU}(5)$ \\
\hline \hline
\end{tabular}

Table II displays the ratios of resulting gaugino masses at the tree level as they arise when $F_{\Phi}$ belongs to the above-mentioned representations of $\mathrm{SO}(10)$ or singlet [14]. The resulting 1-loop relations at the electroweak scale are also displayed. These values and the resulting

TABLE II: Ratios of the gaugino masses at the GUT scale in the normalization $M_{3}(G U T)=1$, and at the electroweak scale in the normalization $M_{3}(E W)=1$.

\begin{tabular}{cccccrrr}
\hline \hline$F_{\Phi}$ & $H$ & $M_{1}^{\mathrm{G}}$ & $M_{2}^{\mathrm{G}}$ & $M_{3}^{\mathrm{G}}$ & $M_{1}^{E W}$ & $M_{2}^{E W}$ & $M_{3}^{E W}$ \\
\hline $\mathbf{1}$ & & 1 & 1 & 1 & 0.14 & 0.29 & 1 \\
$\mathbf{5 4}$ & $\mathrm{SU}(4) \times \mathrm{SU}(2) \times \mathrm{SU}(2)$ & -1 & -1.5 & 1 & -0.15 & -0.44 & 1 \\
$\mathbf{5 4}$ & $\mathrm{SU}(2) \times \mathrm{SO}(7)$ & 1 & $-7 / 3$ & 1 & 0.15 & -0.68 & 1 \\
$\mathbf{2 1 0}$ & $\mathrm{SU}(5) \times \mathrm{U}(1)$ & $-96 / 25$ & 1 & 1 & -0.56 & 0.29 & 1 \\
\hline \hline
\end{tabular}

relic densities can be compared with the universal and nonuniversal representations resulting in the $\mathrm{SU}(5) \mathrm{GUT}$ model $[21,22]$. Since we assume breaking at one scale, the universal model with which we should compare in the $\mathrm{SO}(10) \mathrm{GUT}$ is similar to the universal model in the $\mathrm{SU}(5) \mathrm{GUT}$. In the nonuniversal representations, the relations between gaugino masses change. Thus, e.g. , the 54-dimensional Pati-Salam model of SO(10) may seem at first glance rather similar to $\mathbf{2 4}$ of $\mathrm{SU}(5)$, but we will see that the twice as large bino component has a large effect to the relic density. The bino and wino mass parameters affect directly the lightest neutralino mass and properties. They also affect the value of the $\mu$-parameter through the renormalization group equations (RGE) and 
the radiative electroweak symmetry breaking (rEWSB), therefore controlling also the Higgsino component in the lightest neutralino. Since the lightest neutralino mass limit can be deduced from the chargino mass limit, the nonuniversal gaugino masses change the lower limit for the neutralino mass: for $\mathbf{5 4}$ the neutralino mass limit is smaller than in the universal case, while for $\mathbf{2 1 0}$ the mass limit is close to the chargino mass limit.

\section{B. Calculation of Dark Matter Relic Density}

We calculate the SUSY spectrum for each model with the program SOFTSUSY (version 2.0.11) [23], and the resulting relic density with the program micrOMEGAs (version 2.0.7) [24, 25, 26]. For the relic density, we use here the WMAP combined 3 yr limits [1]

$$
\Omega_{C D M} h^{2}=0.11054_{-0.00956}^{+0.00976}(2 \sigma) .
$$

In all the figures that we show below, the filling denoted by wmap is the WMAP-preferred region. For the $b \rightarrow s \gamma$ experimental branching fraction, we have used the two sigma world average [27],

$$
B R(b \rightarrow s \gamma)=\left(355 \pm 24_{-10}^{+9} \pm 3\right) \times 10^{-6} .
$$

The areas enclosed by the bsg contour are disallowed by the $b \rightarrow s \gamma$ constraint. For the particle masses, the following limits are applied [26]: $m_{\tilde{e}_{R}}>99.4$ or $100.5 \mathrm{GeV}$ depending on if the lightest neutralino mass is below or above $40 \mathrm{GeV}, m_{\tilde{\mu}_{R}}>95 \mathrm{GeV}, m_{\tilde{\tau}_{1}}>80.5$ to $88 \mathrm{GeV}$ depending on the lightest neutralino mass (from 10 to 75 $\mathrm{GeV}), m_{\tilde{\nu}_{i}}>43 \mathrm{GeV}$, and $m_{\tilde{\chi}^{ \pm}}>73.1$ to $103 \mathrm{GeV}$ depending on the sneutrino masses (from 45 to $425 \mathrm{GeV}$ ). In the figures, lep shows an area where the experimental mass limits are not met, rge shows an area where there is no radiative EWSB, and Isp the area where neutralino is not the LSP. The curve $m_{h}=114 \mathrm{GeV}$ is depicted in the figures (dash-dotted line denoted by h). For the shown parameter regions, when otherwise experimentally allowed, Higgs is always heavier than $91 \mathrm{GeV}$, which is the Higgs mass limit in MSSM for $\tan \beta \geq 10$ assuming maximal top mixing [28].

\section{Representation 54}

The area of preferred thermal relic density for the two chains of the $\mathbf{5 4}$ dimensional representation are shown for sets of parameters in Figs. 1, 2, and 3. In each set of three figures, the first figure (a) represents the neutralino relic density for given parameters with collider constraints depicted in the plot, the second figure (b) shows, for the same parameters, the identity of the next-to-lightest supersymmetric particle, and the third figure (c) shows the lightest neutralino composition in RGB-color encoding, (i.e., colors, or hues of black and white, indicate the particle as shown in the figure; therefore the mixture of the colors, or hues of black and white, describes the nature of the $\widetilde{\chi}_{1}^{0}$-composition). In each of the figures, the WMAPpreferred relic density filling is also superimposed to the graph.

As can be seen from the Table II, the lightest neutralino is expected to be bino rather than wino. The large bino component tends to suppress the neutralino annihilation cross section, since bino lacks the s-channel $Z$-boson annihilation mode. A substantial Higgsino component is usually needed to help to increase the annihilation rate, unless there happens to be coannihilation or, e.g. , an open Higgs s-channel annihilation mode available.

In Fig. 1 relic density, the next-to-lightest supersymmetric particle (NLSP) and LSP composition in the breaking chain $\mathrm{SU}(2) \times \mathrm{SO}(7)$ are shown. Because $\tilde{\chi}_{1}^{0}$ is mostly bino, the spectrum with preferred relic density is quite light and conflicts with collider constraints in some parts of the parameter space. With increasing gaugino masses also the Higgsino component in the neutralino LSP increases, and at the point where the change to dominantly Higgsino LSP occurs, also the relic density drops. The overall relic density is not very high, thus allowing a wider WMAP-preferred region than, e.g. , in the singlet, i.e., mSUGRA case [22]. For a given $M_{2}$, the corresponding $M_{1}$ is smaller than in the singlet case, which results in a smaller $\mu$ value at the EW-scale. This has an effect of an increasing the Higgsino component in the lightest neutralino thus boosting the annihilation. The NLSP is chargino, and with increasing Higgsino component it eventually becomes the LSP.

In the allowed region, $M_{2}$ is less than $740 \mathrm{GeV}$, which restricts the lightest chargino mass to values less than $\sim$ $150 \mathrm{GeV}$. The lower limit for the chargino mass is the LEP limit. The partners of the SM fermions are heavier than 300-500 GeV. Thus, assuming that the neutralino is responsible for the dark matter, this breaking chain of the gauge symmetry has as a robust prediction for the upper limit of the chargino mass, and furthermore it is lighter than the squarks and sleptons. For $M_{2} \sim$ $350 \mathrm{GeV}$ the whole neutralino and chargino spectrum, and even the gluino, is lighter than the sfermions for the LEP allowed and WMAP-preferred region. The same is true also for $M_{2} \sim 630 \mathrm{GeV}$ and $m_{0} \gtrsim 940 \mathrm{GeV}$ for the WMAP-preferred region.

The $b \rightarrow s \gamma$ constraint cuts away a considerable area from the otherwise allowed region. Including a 10\% error in theoretical calculations of the decay, the constraints from $b \rightarrow s \gamma$ loosen considerably, and all of the otherwise allowed WMAP area becomes available. The lightest Higgs is always lighter than $114 \mathrm{GeV}$, but heavier than $91 \mathrm{GeV}$.

The effect of varying the universal trilinear coupling $A_{0}$ is shown in Fig. 2. In contrast to the previous figure, here the sign of the $\mu$-parameter is chosen to be negative. In general, giving the $A_{0}$-parameter a nonzero value tends to increase the relic density. Increasing $\left|A_{0}\right|$ will help the $m_{H_{u}}^{2}$ to run larger negative values during the RG- 


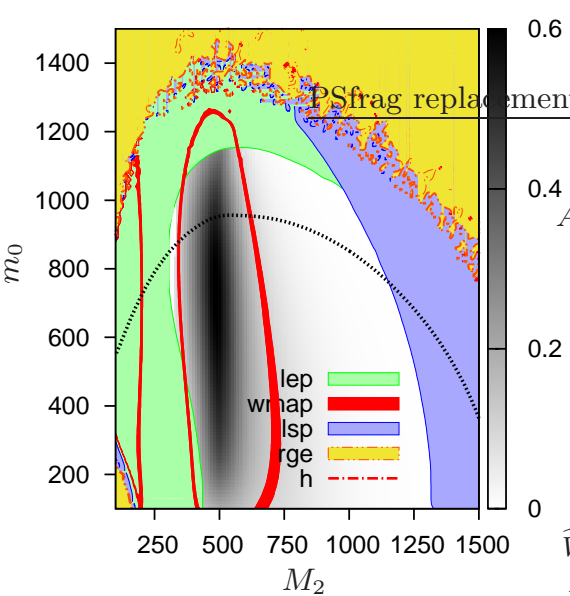

(a) Relic density

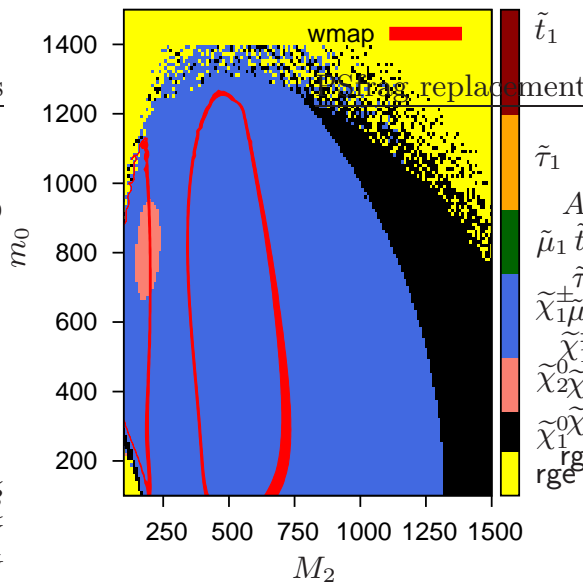

(b) NLSP map

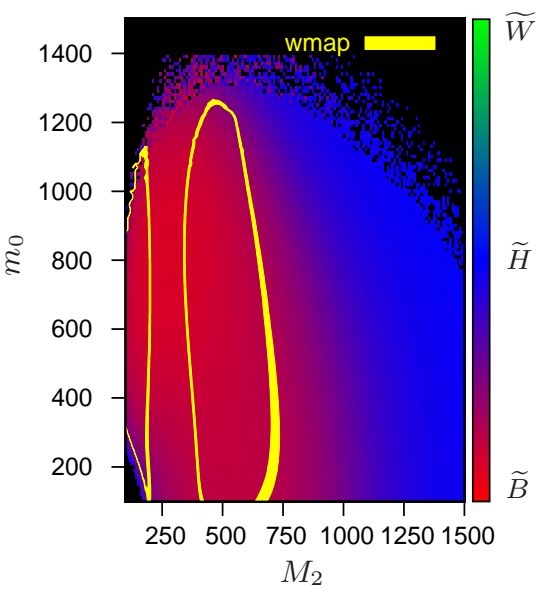

(c) Neutralino composition

FIG. 1: Relic density $\Omega_{\chi} h^{2}$ in the representation 54 with $H=\mathrm{SU}(2) \times \mathrm{SO}(7)$ in the $\left(M_{2}, m_{0}\right)$ plane for $\tan \beta=10, \operatorname{sgn}(\mu)=+1, A_{0}=0$. In 1a the dark shaded areas represent the larger relic density. The filling denoted by wmap is the WMAP preferred region, lep shows an area, where the experimental mass limits are not met, rge shows an area where there is no radiative EWSB, and Isp the area where neutralino is not the LSP. $\mathrm{h}$ encloses the area with $m_{h}<114 \mathrm{GeV}$, and in the following figures bsg the area disallowed by $b \rightarrow s \gamma$ limits. In $1 b$ the NLSP within the same region is plotted, and in $1 \mathrm{c}$ the $\widetilde{\chi}_{1}^{0}$ composition.

evolution, and therefore to increase the actual (absolute) value for the $\mu$-parameter via the rEWSB. This in turn favors the bino component in the $\widetilde{\chi}_{1}^{0}$ composition in the $\mathrm{SU}(2) \times \mathrm{SO}(7)$ chain. The effect can be seen in the LSPcomposition Fig. 2c. The preferred WMAP region still follows the transition zone of the $\widetilde{\chi}_{1}^{0}$ from bino to Higgsino. The effect of negative $\mu$ is most visible in the fact that the NSLP in this case is $\widetilde{\chi}_{2}^{0}$, since negative $\mu$ tends to increase the lightest chargino mass (at least in the limit of $\left.\left|M_{2}\right|<|\mu|\right)$. The $b \rightarrow s \gamma$ constrains an area with negative $A_{0}$ values at $M_{2}<1 \mathrm{TeV}$, and again including also the theoretical error, the constrained area becomes considerably smaller. The light Higgs boson mass is heavier than $114 \mathrm{GeV}$ above the dash-dotted line.

In Fig. 3 the relic density, NLSP and $\widetilde{\chi}_{1}^{0}$ composition are plotted for the $\mathrm{SU}(4) \times \mathrm{SU}(2) \times \mathrm{SU}(2)$ breaking chain. In this breaking chain the $\left|M_{1}\right|$ and $\left|M_{2}\right|$ values are closer to each other at the EW-scale than in the $\mathrm{SU}(2) \times \mathrm{SO}(7)$ chain, but still wider spread than in the singlet. That has an effect of increasing the $\mu$ value, therefore resulting in a smaller Higgsino component for the equal $M_{2}$ values for the two chains of the $\mathbf{5 4}$ dimensional representation. Again the WMAP allowed narrow region follows the transition from the $\widetilde{\chi}_{1}^{0}$ from bino to Higgsino. The $b \rightarrow s \gamma$ and Higgs boson $114 \mathrm{GeV}$ limits cut pieces from near the low $M_{2}, m_{0}$-values. The NLSP is mostly the lightest chargino, but an interesting region exists with small $m_{0}$ near the area, where $\widetilde{\chi}_{1}^{0}$ is no longer the LSP; along the line of transition from the stau NLSP to the smuon NLSP there is a narrow region, where $\widetilde{\chi}_{1}^{0}$, stau and smuon masses are very close to each other, and the coannihilations may reduce the relic density to an acceptable level. For example, for $M_{2}=1400 \mathrm{GeV}$, the LSP mass is around $410 \mathrm{GeV}$, and for $M_{2}=1000 \mathrm{GeV}$, the LSP mass is $290 \mathrm{GeV}$ for the WMAP-preferred area. However, this area is highly prone to numerical subtleties, and the ordering of the LSP identity changes in the preferred relic density range, when comparing the output of different spectrum calculators. Reducing the $m_{0}$ parameter further makes the stau become the LSP. From the collider point of view, such regions may be especially interesting, as they would lead to quasistable smuons or staus.

\section{Representation 210}

In the representation $\mathbf{2 1 0}$ we inspected the breaking chain through the intermediate gauge group $\mathrm{SU}(5) \times \mathrm{U}(1)$, called flipped $\mathrm{SU}(5)[29,30,31,32]$. In Fig. 4a the area of preferred thermal relic density in the representation $\mathbf{2 1 0}$ is plotted for a set of (GUT scale) parameters. For the chosen parameters, rather large WMAP-preferred regions are found for large values of $M_{2}$ and/or $m_{0}$ parameters. When not Higgsino, the lightest neutralino is expected to be wino, rather than bino (see Table II), and therefore the neutralino relic density to be very small. In general, due to the wino being the smallest of the two electroweak gaugino parameters, it characterizes the lightest neutralino. Since the lightest chargino is characterized also by this parameter, for a large part of the parameter space, the masses of the $\widetilde{\chi}_{1}^{0}$ and $\widetilde{\chi}_{1}^{ \pm}$are very close to each other, which boosts the rapid neutralino relic density annihilation. The situation resembles the one arising in the anomaly mediated SUSY breaking scenario, where also both the lightest neutralino and chargino are characterized by the wino mass param- 


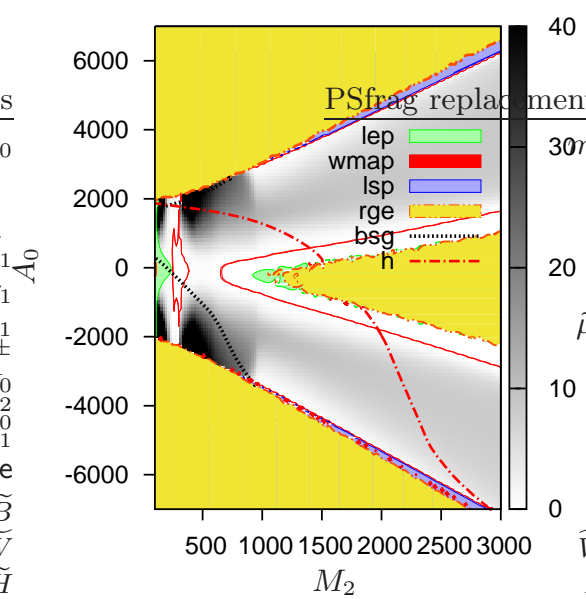

(a) Relic density

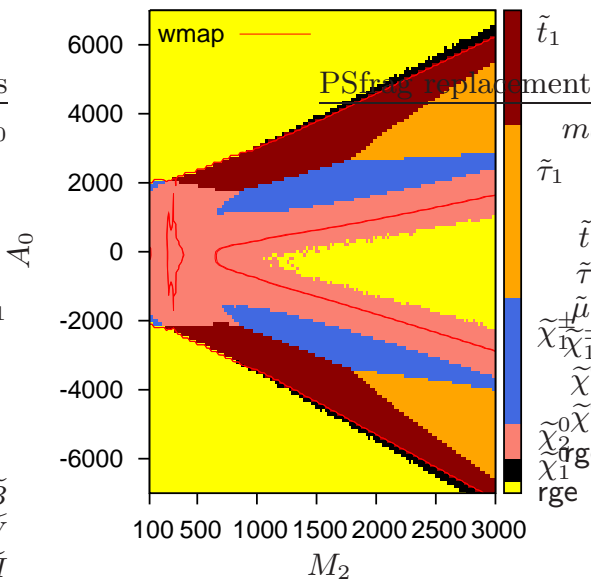

(b) NLSP map

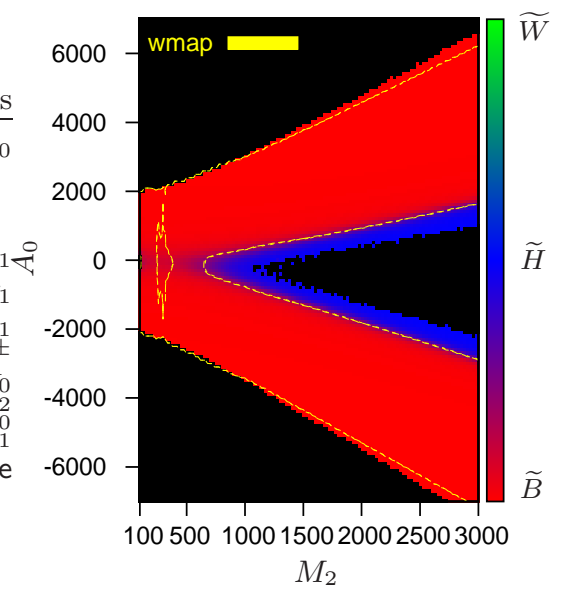

(c) Neutralino composition

FIG. 2: Representation 54: $H=\mathrm{SU}(2) \times \mathrm{SO}(7), \tan \beta=10, \operatorname{sgn}(\mu)=-1, m_{0}=1$ TeV. Otherwise as in Fig. 1 .

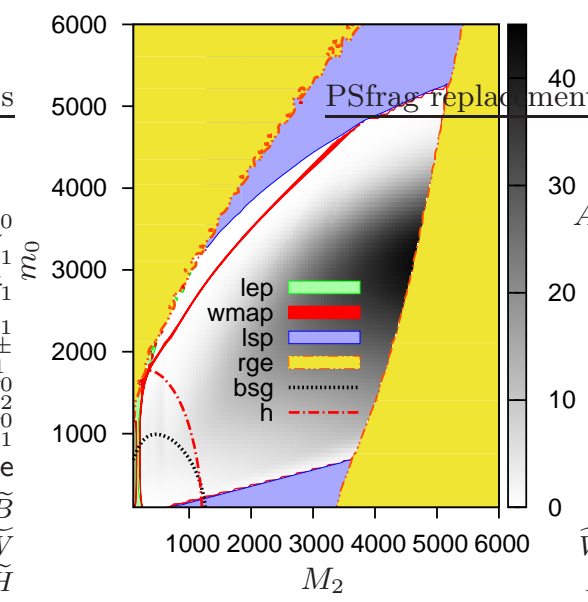

(a) Relic density

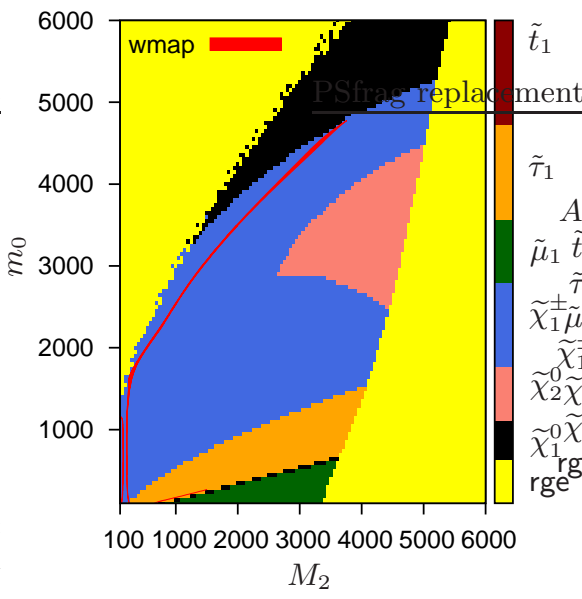

(b) NLSP map

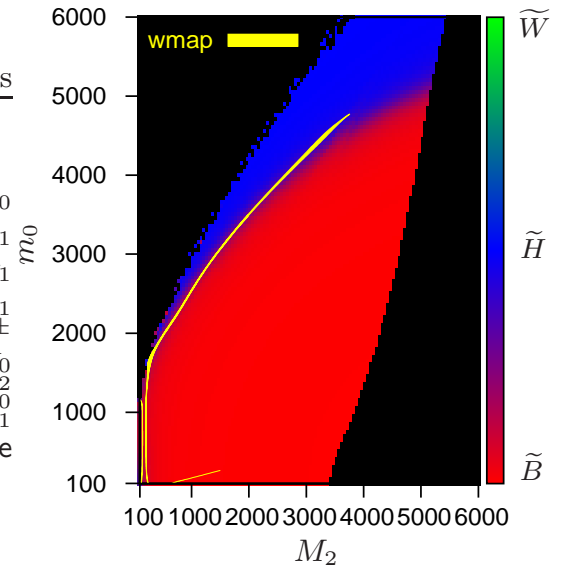

(c) Neutralino composition

FIG. 3: Representation 54 with $H=\mathrm{SU}(4) \times \mathrm{SU}(2) \times \mathrm{SU}(2)$ in the $\left(M_{2}, m_{0}\right)$ plane for $\tan \beta=10, \operatorname{sgn}(\mu)=+1, A_{0}=0$. Otherwise as in Fig. 1.

eter.

Since both the wino and Higgsino have a large annihilation cross section, the WMAP-preferred relic density region does not have to follow the transition zone of $\widetilde{\chi}_{1}^{0}$ from wino to Higgsino. The increase of the relic density to the observed level is mainly due to the increase of the mass parameters $M_{2}$ and $m_{0}$. An interesting change in the pattern can be seen on the diagonal of the figures, where the $\widetilde{\chi}_{1}^{0}$ and $\widetilde{\chi}_{1}^{ \pm}$, and also at some point $\widetilde{\chi}_{2}^{0}$, masses are very close to each other leading to enhanced coannihilation through the processes $\widetilde{\chi}_{1}^{0} \widetilde{\chi}_{1}^{ \pm} \rightarrow q_{u} \bar{q}_{d}$ and $\widetilde{\chi}_{1}^{0} \widetilde{\chi}_{1}^{0}, \widetilde{\chi}_{1}^{ \pm} \widetilde{\chi}_{1}^{ \pm} \rightarrow q \bar{q}, \bar{\ell}, W^{+} W^{-}$, which allows the acceptable parameter space to extend to very large values of $M_{2}$ and $m_{0}$.

The WMAP-preferred region is very wide as compared to, e.g. , the universal model or other models in SU(5) $[22,33]$. The spectrum is relatively heavy for the WMAPpreferred region, around a couple of $\mathrm{TeV}$. This leads to a wide range in the parameter space, but from the point of view of the coming Large Hadron Collider such a mass spectrum may be problematic. However, the representation $\mathbf{2 1 0}$ produces naturally a neutralino with mass around a $\mathrm{TeV}$, which seems to be favorable in the view of the recent PAMELA [34] and ATIC [35] results of the excess positron and positron+electron flux. Under certain circumstances, a nearby clump of 600-1000 GeV neutralino LSP could fit into these observations [36].

In Fig. 5 the area of preferred thermal relic density in the representation $\mathbf{2 1 0}$ is plotted for the same set of (GUT scale) parameters as in Fig. 4, except that now the trilinear $A_{0}$-parameter is varied along the y-axis, and the $m_{0}$ is set to $1 \mathrm{TeV}$. The interesting feature in this figure is the existence of the pseudoscalar Higgs annihilation channel through the $M_{2}$-values. This reduces greatly the relic density in the parameter space where the LSP mass equals or is less than half of the $A$-Higgs mass. 


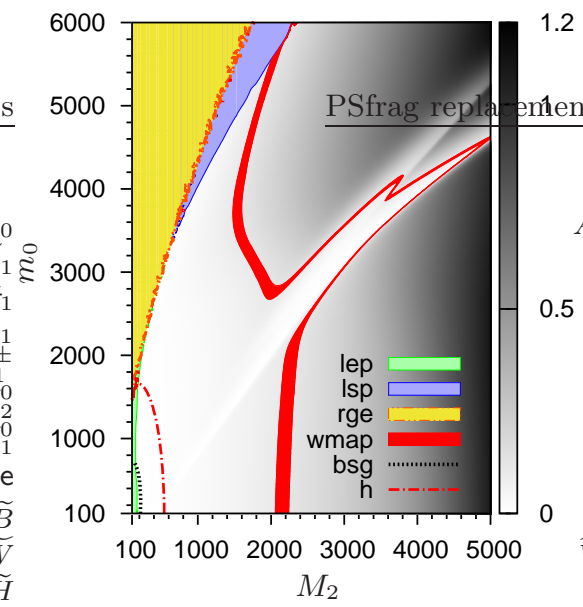

(a) Relic density

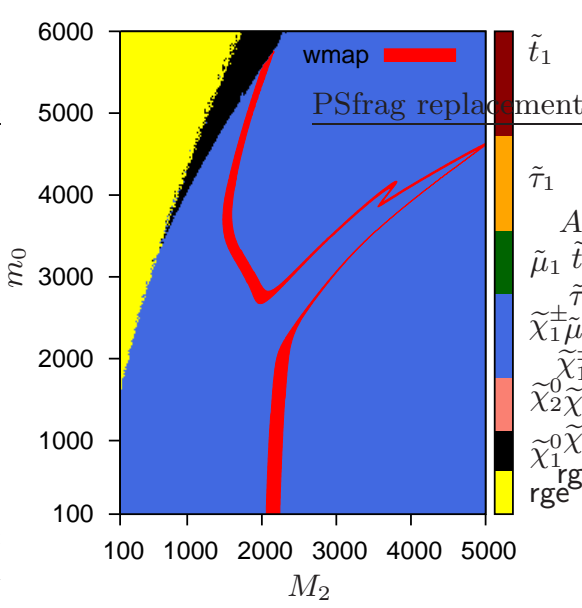

(b) NLSP map

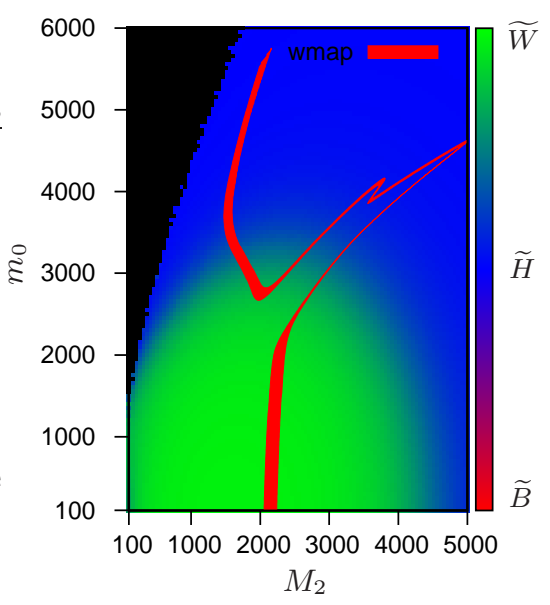

(c) Neutralino composition

FIG. 4: Representation 210: $\tan \beta=10, \operatorname{sgn}(\mu)=+1, A_{0}=0$. Otherwise as in Fig. 1.

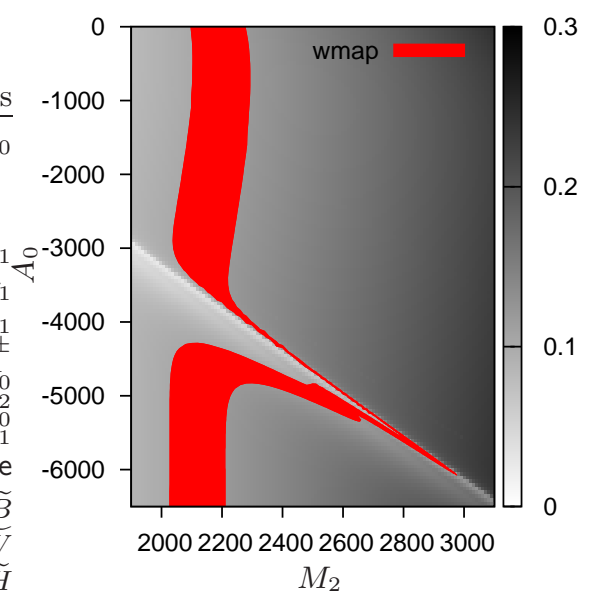

FIG. 5: Relic density in Rep 210:

$\tan \beta=10, \operatorname{sgn}(\mu)=+1, m_{0}=1 \mathrm{TeV}$. The collider constraints are fulfilled.

This has the effect of pushing the WMAP-preferred relic density region to heavier neutralino masses, and therefore to larger $M_{2}$ values. The width of the WMAP region is naturally the same as before, since the top of the figure, where $A_{0}=0$, coincides with Fig. 4 with $m_{0}=1000 \mathrm{GeV}$. With these parameters, the NLSP is always the lighter chargino, and the lightest neutralino is dominantly a wino, which can also be read from Fig. 4c.

\section{DISCUSSION AND SUMMARY}

We studied the dark matter allowed regions in the $\mathrm{SO}(10)$ GUT representations, of which all but the singlet may lead to nonuniversal gaugino masses. The WMAPpreferred relic density regions are quite distinct for different representations, thus leading to quite different particle spectra for each representation.
In the representation $\mathbf{5 4}$, the lightest neutralino is predominantly a bino, leading to the narrow areas of the WMAP-favored region. The excessive relic density is diluted either by increasing the Higgsino component or by coannihilation with other particles. The breaking chain $\mathrm{SU}(2) \times \mathrm{SO}(7)$ predicts an upper limit for the lighter chargino mass for the chosen parameters. For part of the WMAP allowed region, the whole neutralino and chargino spectrum is lighter than the spectrum of sfermions. For the $\mathrm{SU}(4) \times \mathrm{SU}(2) \times \mathrm{SU}(2)$ breaking chain, the relic density area is narrow in the parameter space. Interestingly, there may exist a region, where the stau, smuon and the lightest neutralino masses are in a very close range to each other. This can lead to long-lived staus and smuons, which may be stable in the collider time scale.

In the $\mathbf{2 1 0}$ dimensional representation the lightest neutralino is either wino or Higgsino, which leads to a low thermal relic density. In addition, the lightest chargino and the lightest neutralino tend to be close in mass, thus providing a coannihilation channel. The preferred relic density area is quite large. The sparticle spectrum is heavy, as compared to the universal mSUGRA case. Only in a small part of the WMAP-preferred parameter region are a few SUSY particles expected to be within the kinematic reach of the LHC.

In this work we have studied each representation separately. It is obvious that if several representations affect simultaneously the composition of neutralinos, the possible WMAP-preferred region in the parameter space may be relaxed. However, if the neutralinos and charginos are found with a certain mass pattern, it helps to understand the relation of the lightest neutralino with dark matter, if characteristics of each representation are known. 


\section{ACKNOWLEDGMENTS}

The work of K.H. is supported by the Academy of Finland (Project No. 115032). The work of J.L. is supported by the Bundesministerium für Bildung und Forschung, Berlin-Bonn.
[1] D. N. Spergel et al. (WMAP), Astrophys. J. Suppl. 170, 377 (2007), astro-ph/0603449.

[2] K. Huitu, J. Laamanen, P. N. Pandita, and S. Roy, Phys. Rev. D72, 055013 (2005), hep-ph/0502100.

[3] H. Fritzsch and P. Minkowski, Ann. Phys. 93, 193 (1975).

[4] Z.-Y. Zhao, J. Phys. G8, 1019 (1982).

[5] M. Drees and J. M. Kim (2008), 0810.1875.

[6] S. Bhattacharya, A. Datta, and B. Mukhopadhyaya, JHEP 10, 080 (2007), 0708.2427.

[7] B. Ananthanarayan and P. N. Pandita, Int. J. Mod. Phys. A22, 3229 (2007), 0706.2560.

[8] R. N. Mohapatra and G. Senjanovic, Phys. Rev. Lett. 44, 912 (1980).

[9] B. Bajc, G. Senjanovic, and F. Vissani, in ${ }^{*} B u-$ dapest 2001, High energy physics* hep2001/198 (2001), hep-ph/0110310.

[10] S. Dimopoulos and F. Wilczek, in Proceedings of the 19th Course of the International School of Subnuclear Physics, Erice, Italy, 1981, edited by A. Zichichi (Plenum Press, New York, 1983).

[11] E. Cremmer, S. Ferrara, L. Girardello, and A. Van Proeyen, Phys. Lett. B116, 231 (1982).

[12] S. Ferrara, L. Girardello, and H. P. Nilles, Phys. Lett. B125, 457 (1983).

[13] R. Slansky, Phys. Rept. 79, 1 (1981).

[14] N. Chamoun, C.-S. Huang, C. Liu, and X.-H. Wu, Nucl. Phys. B624, 81 (2002), hep-ph/0110332.

[15] G. F. Giudice and R. Rattazzi, Nucl. Phys. B511, 25 (1998), hep-ph/9706540.

[16] C. S. Aulakh and S. K. Garg (2008), 0807.0917.

[17] C. S. Aulakh and S. K. Garg, Nucl. Phys. B757, 47 (2006), hep-ph/0512224.

[18] B. Bajc, I. Dorsner, and M. Nemevsek, JHEP 11, 007 (2008), 0809.1069.

[19] C. S. Aulakh and R. N. Mohapatra, Phys. Rev. D28, 217
(1983).

[20] T. Fukuyama, A. Ilakovac, T. Kikuchi, S. Meljanac, and N. Okada, J. Math. Phys. 46, 033505 (2005), hep-ph/0405300.

[21] S. F. King, J. P. Roberts, and D. P. Roy (2007), arXiv:0705.4219.

[22] K. Huitu et al., Eur. Phys. J. C58, 591 (2008), 0808.3094.

[23] B. C. Allanach, Comput. Phys. Commun. 143, 305 (2002), hep-ph/0104145.

[24] G. Belanger, F. Boudjema, A. Pukhov, and A. Semenov, Comput. Phys. Commun. 149, 103 (2002), hep-ph/0112278.

[25] G. Belanger, F. Boudjema, A. Pukhov, and A. Semenov, Comput. Phys. Commun. 174, 577 (2006), hep-ph/0405253.

[26] G. Belanger, F. Boudjema, A. Pukhov, and A. Semenov, Comput. Phys. Commun. 176, 367 (2007), hep-ph/0607059.

[27] E. Barberio et al. (Heavy Flavor Averaging Group (HFAG)) (2007), 0704.3575.

[28] Search for charged Higgs bosons: Preliminary combined results using LEP data collected at energies up to 209GeV (2001), hep-ex/0107031, hep-ex/0107031.

[29] A. De Rujula, H. Georgi, and S. L. Glashow, Phys. Rev. Lett. 45, 413 (1980).

[30] S. M. Barr, Phys. Lett. B112, 219 (1982).

[31] J. P. Derendinger, J. E. Kim, and D. V. Nanopoulos, Phys. Lett. B139, 170 (1984).

[32] I. Antoniadis, J. R. Ellis, J. S. Hagelin, and D. V. Nanopoulos, Phys. Lett. B194, 231 (1987).

[33] K. Huitu, J. Laamanen, and S. Roy (2007), 0710.2058.

[34] O. Adriani et al. (2008), 0810.4994.

[35] J. Chang et al., Nature 456, 362 (2008).

[36] D. Hooper, A. Stebbins, and K. M. Zurek (2008), 0812.3202 . 